\title{
Galactic cosmic ray modulation in the heliosphere based on muon telescopes and ion chambers data
}

\section{R. Modzelewska ${ }^{a *}$ and H.S. Ahluwalia $b$}

a Faculty of Exact and Natural Sciences, Institute of Mathematics, Siedlce University, Konarski Str. 2 08-110 Siedlce, Poland

b Physics and Astronomy Department, University of New Mexico, Albuquerque, NM 87131, USA

E-mail: renatam@uph.edu.pl, hsa@unm.edu

We study the galactic cosmic ray modulation in the heliosphere based on muon telescopes and ion chambers data. We analyze the modulation parameters of galactic cosmic ray transport in the heliosphere retrieved from GCR anisotropy for solar cycles 18-23 and parts of 17 and 24, covering the period 1937-2018. It was found that the ratio $\alpha$ of mean free paths normal and parallel to mean interplanetary magnetic field $\mathbf{B}$ is polarity dependent at Earth orbit with higher values for periods around solar minima for positive polarity $(A>0)$ than for negative $(A<0)$. Timeline of ratio $\alpha$ for more than 7 decades, exhibits a slight $\sim 11$-year and dominant $\sim 22$-year variation and has a strongly polarity dependent character with the considerable enhancement in the minimum epoch of solar activity for $\mathrm{A}>0$ magnetic polarity. Results will be confronted with current modulation theories. We examine the diffusion-convection implications and the solar cycle and solar magnetic polarity dependence of cosmic ray modulation for muon and ion chamber data.

37th International Cosmic Ray Conference (ICRC 2021)

July 12th-23rd, 2021

Online-Berlin, Germany

\section{*Presenter}




\section{Introduction}

Transport of galactic cosmic ray (GCR) particles in the heliosphere is controlled by the expansion of the solar wind and frozen-in interplanetary magnetic field (IMF). The global modulation of GCR in the heliosphere is governed by four most important processes: convection, diffusion, particle drifts (due to gradient and curvature of the IMF, and on the heliospheric current sheet (HCS)), and adiabatic cooling. Recently, despite significant progress in our understanding of the GCR modulation by the solar wind, e.g. [1], up to now there remains some difficulties in insufficient knowledge of the spatial, rigidity and especially the temporal dependence of the diffusion coefficients. However, for selecting an appropriate set of modulation parameters used in theoretical modelling (especially diffusion coefficients, etc.), one criterion remains the most important (if it is possible), to estimate them from the experimental data. We analyze long-period variations of the radial and azimuthal components of the GCR anisotropy for the period of 19372018 found by muon telescopes (MT) and ion chambers (IC) data. Observations of GCR anisotropy by MT and IC can be successfully used to calculate of various parameters characterized modulation of GCR by the solar wind. A general purpose of this paper is to estimate the time variation of parameter $\alpha$, being the ratio of mean free paths normal and parallel to mean IMF $\mathbf{B}$.

\section{Experimental Data}

GCR solar diurnal anisotropy observed at Earth is a consequence of equilibrium state between inward diffusion of GCR and outward radial convection by the solar wind. The convectiondiffusion mechanism of GCR diurnal variation were proposed by [2-4].

Here we present the timeline of $\boldsymbol{\alpha}$ using yearly solar diurnal variations recorded by Forbush [5] shielded IC at Cheltenham for 1937-1977, MT data at Nagoya for 1971-2017, details please see in [6-7] and vertical MT Hobart (Australia) for 2007-2018, location 43.0S, 147.3E, 65 m, median primary rigidity of response $\mathrm{Rm}=54.6 \mathrm{GV},[8]$.

\section{Data analysis}

Ahluwalia and Dorman [9] derived the following relations for $A r, A \varphi$ assuming flat HCS:

$$
\begin{aligned}
& \left(A r^{p}+A r^{n}\right) / 2=3 / v(C V-K r r G r)(1), \quad K r r=K_{\|} \cos ^{2} \psi+K_{\perp} \sin ^{2} \psi \\
& \left(A \varphi^{p}+A \varphi^{n}\right)=(1-\alpha) \lambda_{\|} G r \sin 2 \psi \quad(2), \quad \lambda_{\|} G r=-A r+\frac{3 C V}{v}+A \varphi \operatorname{Tan} \psi
\end{aligned}
$$

the superscripts $\mathrm{p} / \mathrm{n}$ apply to positive and negative $\mathbf{B}$ polarities, $G r$ is GCR radial density gradient directed away from the Sun, $K r r$ is radial diffusion coefficient, $\lambda_{\|}$is the free path parallel to $\mathbf{B}, \boldsymbol{V}$ is solar wind velocity, $v(\sim \mathrm{c})$ is GCR velocity, $\psi$ is IMF spiral angle, its yearly value fluctuates about $45^{\circ}, C \approx 1.5$ is Compton-Getting coefficient.

Yearly $\boldsymbol{\alpha}$ values are obtained as follows from (2):

$\boldsymbol{\alpha}=1-\left(A \varphi^{p}+A \varphi^{n}\right) /\left(\lambda_{\|} G r \sin 2 \psi\right)$ 


\section{Results}

We analyzed the modulation parameters of galactic cosmic ray transport in the heliosphere retrieved from GCR anisotropy for solar cycles 18-23 and parts of 17 and 24, covering the period 1937-2018. Fig. 1 presents the timeline of parameter $\alpha$ for IC Cheltenham for 1937-1977, MT Nagoya for 1971-2017, details please see in [6-7] and MT Hobart for 2007-2018 [8].

We found that the ratio $\alpha$ of mean free paths normal and parallel to mean IMF $\mathbf{B}$ is polarity dependent at Earth orbit with higher values for periods around solar minima for positive polarity $(A>0)$ than for negative $(A<0)$. Timeline of modulation parameter $\alpha$ for more than 7 decades, exhibits a slight $\sim 11$-year and dominant $\sim 22$-year variation and has a strongly polarity dependent character with the considerable enhancement in the minimum epoch of solar activity for $A>0$ IMF magnetic polarity.

Here we did not include contributions from drift-related symmetric (normal to HCS) gradient $\left(G_{\theta s}\right)$. Simone et al. [10] state its value measured for low energy protons by Ulysses and PAMELA is smaller than expected from theories but signs are correct; supporting earlier Simpson et al. [11] inference that latitude gradients measured by Ulysses are small. Minnie et al. [12] argue that particle drifts may be suppressed by turbulence in background B. Engelbrecht et al. [13] further discuss reduction of drift coefficients in the presence of turbulence.

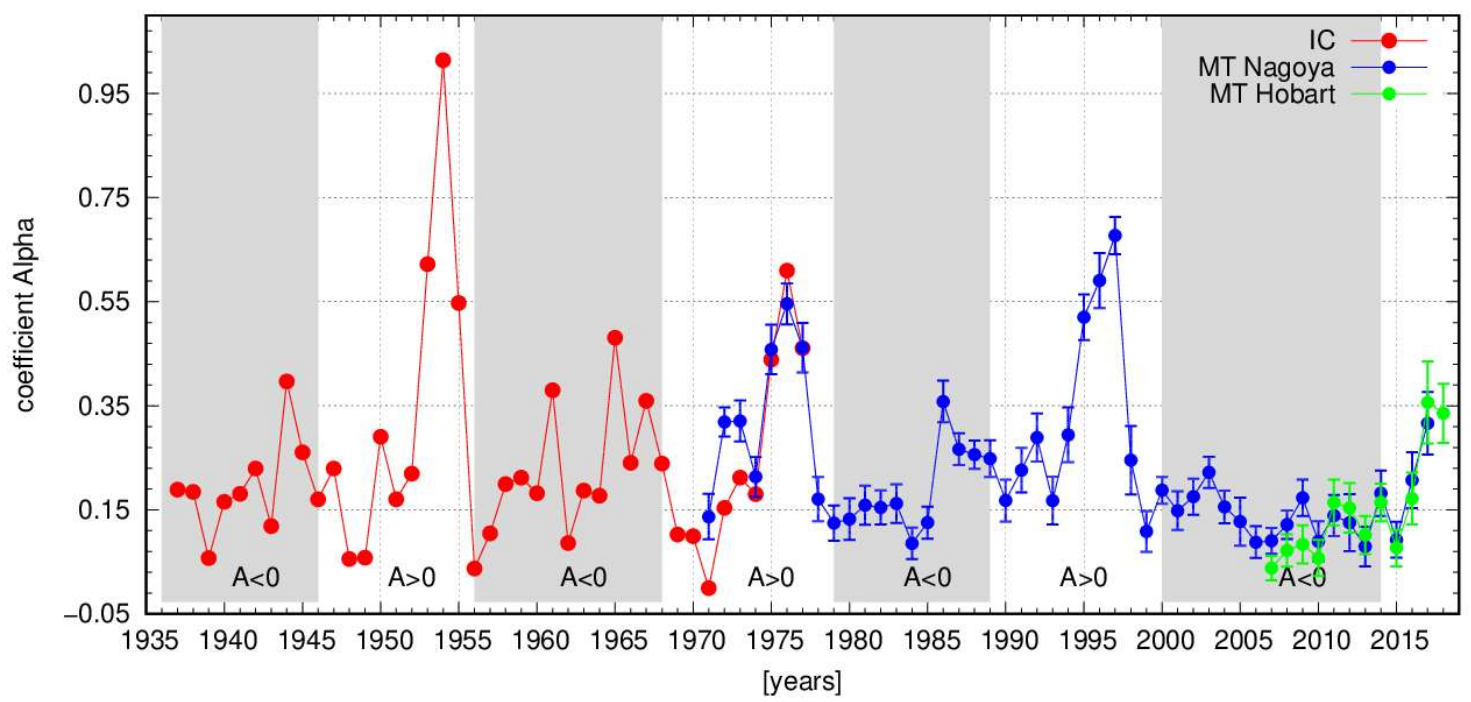

Fig. 1. Yearly $\boldsymbol{\alpha}$ for IC Cheltenham, MT Nagoya and MT Hobart data for 1937-2018, for solar cycles $18-23$ and parts of 17 and 24 .

\section{References}

[1] M. S. Potgieter, Living Rev. Solar Phys. 10 (2013) 3-66

[2] H. S. Ahluwalia and A.J. Dessler, Planet. Space Sci. 9 (1962) 195-210

[3] G. F. Krymsky, Geomagnetism \&Aeronomy 4, 6, (1964) 977-986 (in Russian)

[4] E. N. Parker, Planet. Space Sci. 12 (1964) 735-749

[5] S. E. Forbush, Time Variations of Cosmic Rays, Handbuch der Physik, v. XLIX/1, Geophys. III, Part 1. Ed. S. Flugge, Springer Verlag, New York, (1966) 159-247 
[6] H. S. Ahluwalia and R. Modzelewska, Advances in Space Research 66, 2 (2020) 462-467

[7] H. S. Ahluwalia and R. Modzelewska, Advances in Space Research 68, 3 (2021) 1502-1506

[8] Y. Okazaki et al. ApJ. 681 (2008) 693-707

[9] H. S. Ahluwalia and L. I. Dorman, J. Geophys. Res. 102 (1997) 17433-17443

[10] N. De Simone, V. Di Felice, J. Gieseler, et al. Astrophys. Space Sci. Trans. 7 (2011) 425-434

[11] J. A.Simpson, J. D. Anglin, V. Bothmer et al. Science 268 (1995) 1019-1023

[12] J. Minnie, J.W. Bieber, W.H. Matthaeus, R.A. Burger, ApJ. 670 (2007) 1149-1158

[13] N. E. Engelbrecht, R. D. Strauss, J. A. le Roux, and R. A. Burger, ApJ. 841 (2017) 107 (8pp) 